\title{
Exogenous HMGN2 inhibits the migration and invasion of osteosarcoma cell lines
}

\author{
Enjie Xu\#, Heng Jiang", Tao Lin, Yichen Meng, Xiao Ma, Jia Yin, Jun Ma, Xuhui Zhou \\ Department of Orthopedics, Changzheng Hospital, Second Military Medical University, Shanghai 200003, China \\ Contributions: (I) Conception and design: E Xu, H Jiang, J Ma, X Zhou; (II) Administrative support: E Xu, H Jiang; (III) Provision of study materials \\ or patients: E Xu, H Jiang, T Lin; (IV) Collection and assembly of data: E Xu, H Jiang, T Lin; (V) Data analysis and interpretation: E Xu, H Jiang, T \\ Lin, Y Meng; (VI) Manuscript writing: All authors; (VII) Final approval of manuscript: All authors. \\ \#These authors contributed equally to this work. \\ Correspondence to: Xuhui Zhou; Jun Ma. Department of Orthopedics, Changzheng Hospital, Second Military University, Shanghai 200003, China. \\ Email: xhzhouspine@163.com; jmaspine@163.com.
}

\begin{abstract}
Background: Osteosarcoma (OS) is among the most prevalent forms of malignant tumors seen in children and teenagers. Early metastasis is a hallmark of OS, and it is therefore important to find new and more effective treatment targets to improve the survival time of patients with the disease. High mobility group $\mathrm{N}$ (HMGNs) is a family of proteins that contributes to the development of a number of different tumors. In particular, HMGN2 was found in our earlier study to be an anti-tumor factor and was seen to impede the metastasis of OS when it was overexpressed. This study aims to further investigate the potential of HMGN2 in anti-tumor treatment.
\end{abstract}

Methods: We overexpressed HMGN2 in 293FT cells via transfection with recombinant lentiviruses and purified HMGN2 protein with flag tags to treat OS cell lines. The cellular location of exogenous HMGN2 was detected by immunocytochemistry, and wound healing and transwell assays were used to study differences in the rates of migration and invasion of cells between each group.

Results: We found that exogenous HMGN2 enters OS cells in a concentration-dependent manner and inhibits the migration and invasion of OS cells, and exogenous HMGN2 regulates the expression of matrix metalloproteinase 2 (MMP2) and MMP9 in OS cells.

Conclusions: Our results demonstrated that exogenous HMGN2 plays a role in inhibiting OS metastasis, which could act as a basis for new ideas for future anti-tumor therapy research.

Keywords: osteosarcoma (OS); HMGN2; exogenous; antitumor; metastasis

Submitted Sep 25, 2019. Accepted for publication Jan 18, 2020.

doi: $10.21037 /$ tcr.2020.02.25

View this article at: http://dx.doi.org/10.21037/tcr.2020.02.25

\section{Introduction}

Among the most prevalent forms of malignant tumors seen in children and teenagers, osteosarcoma (OS) is a bone disease originating from mesenchymal stem cells $(1,2)$. OS is characterized by early metastasis, and approximately $15-25 \%$ of patients have detectable lung metastases at the time of their diagnosis (3). Tumor resection and nonspecific combination chemotherapy are the preferred modes of treatment for OS patients $(4,5)$. With the advances made in relation to primary tumor treatment, patients with the disease have seen a rise in their long-term survival rate (6); however, for patients with distant metastasis, the survival rate remains very low (7-9). It is essential to find new and more effective treatment targets for OS to improve the survival time of patients.

Abnormal expression of high mobility group nucleosome binding domain 2 (HMGN2) is associated with multiple tumors $(10,11)$. HMGN2 takes on a key role in breast cancer progression (12-14) and inhibits the growth and 
promotion of apoptosis of infected bladder cancer cells (15). HMGN2 has been shown to impede growth and bring about apoptosis in the oral squamous cell carcinoma cell line Tca8113 (16). Our previous studies have shown that overexpression of HMGN2 by lentivirus infection in OS cells inhibits the metastasis of cells (17). However, for the treatment of humans, lentiviruses are not yet considered acceptable.

Some cells can secrete HMGN2 extracellularly (18). We speculated that the released HMGN2 is also capable of inhibiting OS metastasis. In addition, there is currently no research to explain the mechanism of the anti-tumor functions of HMGN2. We aimed to investigate whether exogenous HMGN2 enters cells and inhibits the migration and invasion of OS cells and to explore the anti-tumor mechanism of HMGN2 in OS cells further.

\section{Methods}

\section{Cell culture}

We cultured U-2 OS cells in dulbecco's modified eagle medium (DMEM, Hyclone, Utah, USA) medium containing $10 \%$ fetal bovine serum (FBS, Gibco, NY, USA), penicillin (100 U/L, Invitrogen, NY, USA), and streptomycin (100 mg/L, Invitrogen, NY, USA). The cells were then incubated in a humidified atmosphere of $5 \% \mathrm{CO}_{2}$ at $37^{\circ} \mathrm{C}$.

We cultured Saos-2 OS cells in McCoy's 5a (Invitrogen, NY, USA) medium containing 15\% FBS (FBS, Gibco, NY, USA), penicillin (100 U/L, Invitrogen, NY, USA), and streptomycin (100 mg/L, Invitrogen, NY, USA). The cells were then incubated in a humidified atmosphere of $5 \%$ $\mathrm{CO}_{2}$ at $37{ }^{\circ} \mathrm{C}$. We selected two OS cell lines for subsequent experiments. The experimens carried out on the U-2 OS cells were repeated on the Saos-2 cells. DMEM was also used to culture human epithelial kidney 293 FT (HEK 293 FT) cells, which were also incubated in a humidified atmosphere of $5 \% \mathrm{CO}_{2}$ at $37^{\circ} \mathrm{C}$.

\section{Overexpression of HMGN2 and purification}

Recombinant lentiviruses with HMGN2 overexpression were obtained along with blank lentivirus from Shanghai GeneChem Co., Ltd. (vector: GV492, Ubi-MCS-3FLAGCBh-gcGFP-IRES-puromycin, Shanghai, China). The lentiviruses with overexpression (HMGN2-flag ${ }^{\circ e}$ ) and the blank lentivirus (oeCON) were used to transfect 293FT cell lines.

By way of pre-experiment, we found the optimal multiplicity of infection (MOI) for U-2 OS virus infection to be 10, and MOI for Saos-2 to be 15. Our formal experiments were carried out based on these MOI values. After three days of cell infection, we carried out flow sorting to maximize the proportion of infected cells and proceeded with the cell culturing process with green fluorescence. Once the cells had reached $70-80 \%$ confluence, RNA was extracted, and we then validated overexpression efficiency using RT-qPCR.

After the overexpression efficiency of HMGN2 was confirmed, infected cells were collected, and nucleoprotein was extracted using NE-PER Nuclear and Cytoplasmic Extraction Reagents (Thermo Fisher Scientific, MA, USA). In line with the manufacturer's instructions, we used FLAG M Purification Kit (Sigma-Aldrich, St. Louis, MO, USA) to purify HMGN2-flag protein. The concentration of HMGN2-flag was confirmed by enzyme linked immunosorbent assay (ELISA, ab49763, Abcam, Cambridge, UK).

\section{$R T-q P C R$}

To measure HMGN2, MMP2, MMP9, and MMP16 expression, we applied quantitative real time polymerase chain reaction (RT-qPCR) using SYBR-Premix Ex Taq (Takara, Japan) and the ABI Prism 7900HT sequence detection system (Applied Biosystems, Carlsbad, CA, USA). In line with the manufacturer's instructions, we used TRIzol to carry out total RNA extraction from each group. The transcription of mRNA into cDNA was carried out with the help of a Reverse Transcription kit (Applied Biosystems, Carlsbad, CA). Specific primers were used to amplify the genes, with the human $\beta$-actin gene employed as an endogenous control. We applied the following PCR primer sequences: HMGN2: forward: 5'-CGATTGTCTGCCCA TGTCCT-3', reverse: 5'-GCAGAACGTACCCTGTTC CA-3'; MMP2: forward: 5'-TACAGGATCATTGGCTAC ACACC-3', reverse: 5'-GGTCACATCGCTCCAGACT -3'; MMP9: forward: 5'-TGTACCGCTATGGTTACAC TCG-3', reverse: 5'-GGCAGGGACAGTTGCTTCT-3'; MMP16: forward: 5'-AGCACTGGAAGACGGTTGG-3', reverse: 5'-CTCCGTTCCGCAGACTGTA-3'; $\beta$-actin: forward: 5'-ACCGAGCGCGGCTACAG-3', reverse: 5 ' - CTTAATGTCACGCACGATTTCC-3'. The comparative $\mathrm{Ct}$ method $\left(2^{-\Delta \Delta C t}\right)$ was applied to analyze the 
data. The RT-qPCR experiment was carried out identically three times with the same samples and primers.

\section{Western blot assay}

NE-PER Nuclear Extraction Reagents (Thermo Fisher Scientific, MA, USA) was used to carry out nuclear protein extraction, and a protein extract kit (Beyotime, Shanghai, China) was employed for the extraction of whole proteins. A BCA Protein Assay Kit (Pierce) was used to determine protein concentration. Proteins were run on sodium dodecyl sulfate polyacrylamide gel electrophoresis (SDSPAGE) gels before the transfer of the separated protein bands onto polyvinylidene fluoride (PVDF, Millipore, MA, USA) membranes. Blocking was carried out in 5\% bovine serum albumin (BSA, Sigma-Aldrich, St. Louis, MO, USA). Probing of membranes took place overnight at $4{ }^{\circ} \mathrm{C}$ with the appropriate primary antibody. The antibodies used were as follows: anti-HMGN2 (1:1,000 dilution, ab199679, Abcam, Cambridge, UK), anti-DDDDK (1:1,000 dilution, ab49763, Abcam), anti-MMP2 (1:500 dilution, ab97779, Abcam), anti-MMP9 (1:1,000 dilution, ab76003, Abcam), antiMMP16 (1:500 dilution, ab73877, Abcam), anti-LMNB1 (1:10,000 dilution, ab16048, Abcam), anti- $\beta$-actin (1:10,000 dilution, ab8226, Abcam). Probing of membranes with a horseradish peroxidase-conjugated secondary antibody was carried out for at room temperature for 1 hour. An enhanced chemiluminescence (ECL) detection system (No. 32106, Pierce) was employed to visualize the bands that were immunoreactive. We selected a loading control based on the recommendation of Abcam (http://www.abcam.com/ primary-antibodies/loading-control-guide). We normalized the relative protein levels in different cell lines to Lamin B1 or $\beta$-actin concentration. Each group was the subject of three separate experiments.

\section{Immunocytochemistry}

OS cells were treated with purified HMGN2-flag protein at a concentration of $10 \mu \mathrm{g} / \mathrm{mL}$ for 12 and 24 hours before fixing with $4 \%$ paraformaldehyde for 30 minutes and then permeabilization with $0.1 \%$ Triton X-100 in PBS for a period of 5 minutes. Blocking took place with $1 \%$ BSA for 2 hours. Cells were incubated overnight in the antiDDDDK antibody (1:100 dilution, ab49763, Abcam, Cambridge, UK) at $4{ }^{\circ} \mathrm{C}$. Cell incubation was carried out with secondary antibody (Cell Signaling Technology, MA, USA), and the cells were mounted with DAPI (Sigma-
Aldrich) after being washed with PBS. Cellular localization of HMGN2-flag was observed and photographed using a confocal microscope (Leica, Germany).

\section{Wound bealing assay}

OS cells were treated with purified HMGN2-flag protein at a concentration of $2 \mu \mathrm{g} / \mathrm{mL}$ for 24 hours in the HMGN2flag group and the HMGN2-flag+Ab group. Blocking of HMGN2 was achieved by adding $10 \mu \mathrm{g} / \mathrm{mL}$ antiHMGN2 antibody to medium in the HMGN2-flag+Ab group (ab199679, Abcam, Cambridge, UK). Cells in the $3 \times$ flag peptide group were treated with $3 \times f l a g$ peptide at a concentration of $0.55 \mu \mathrm{g} / \mathrm{mL}$ for 24 hours (the same number of moles as HMGN2-flag, Sigma-Aldrich, St. Louis, MO, USA). Six-well culture plates were used to plate OS cells that had been treated, which were then grown to $90 \%$ confluence. We used a $200 \mu \mathrm{L}$ micropipette tip to create wounds. We monitored cell migration towards the wound and took photographs. Migration rate $=\left(\mathrm{S}_{0 \mathrm{~h}}-\mathrm{S}_{24 \mathrm{~h}}\right.$ or $\left.\mathrm{S}_{48 \mathrm{~h}}\right) /$ $\mathrm{S}_{0 \mathrm{~h}} \times 100 \% . \mathrm{S}_{0 \mathrm{~h}}$ was the distance of the scratch at 0 hour, $\mathrm{S}_{24 \mathrm{~h}}$, or $\mathrm{S}_{48 \mathrm{~h}}$ was the distance at 24 or 48 hours.

\section{Transwell invasion assay}

Four groups of cells were treated in the same manner as the wound healing assay. The invasion of cells was determined in $6.5 \mathrm{~mm}$ Transwell (Coring, NY, USA). The top chamber had a filter coated with $50 \mu \mathrm{L}$ of diluted matrigel and a lower chamber filled with $500 \mu \mathrm{L}$ of DMEM medium containing $10 \%$ FBS (or McCoy's 5a medium containing $15 \%$ FBS). Cells were resuspended in serumfree medium and added into to the top chamber of each plate. Cell culturing took place over 24 hours, and any noninvading cells were removed. Invading cells were fixed with $4 \%$ paraformaldehyde for a period of 30 minutes before staining with Crystal Violet Staining Solution (SigmaAldrich). Invading cell numbers were counted and analysis was carried out.

\section{Statistical analysis}

Each treatment group underwent three separate experiments. Data analysis was carried out using SPSS version 23.0, and differences were considered statistically significant when $\mathrm{P}$ value $<0.05$. All data were presented as the mean $\pm \mathrm{SD}$. Comparisons between two groups were made using the Mann-Whitney $\mathrm{U}$ test, and differences between four or more 
A
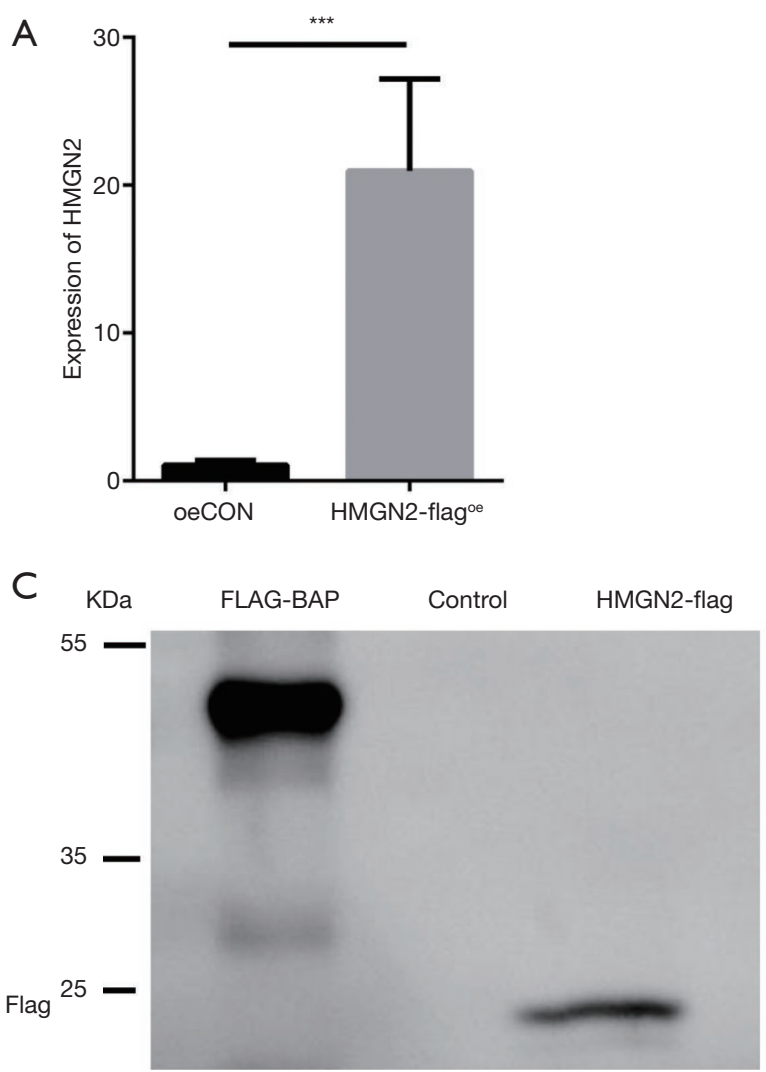

B

KDa Control HMGN2-flag
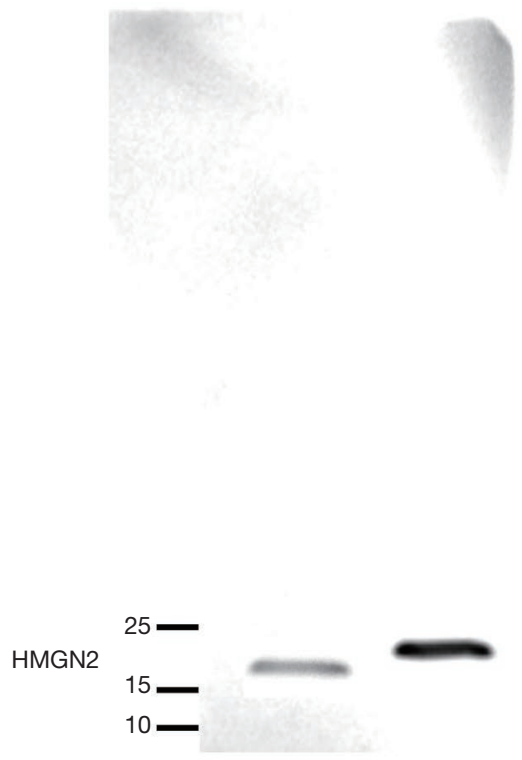

Figure 1 Overexpression and extraction of HMGN2-flag. (A) RT-qPCR showed that the expression level of HMGN2-mRNA in the HMGN2-flag ${ }^{\text {oe }}$ group was significantly higher than in the oeCON group. (B) Purification of HMGN2-flag and detection by Western blot assay. HMGN2 was detected in purified protein using an anti-HMGN2 antibody. Control: nuclear protein extracts of oeCON group; HMGN2-flag: a purified protein with flag tag from HMGN2-flag ${ }^{\text {oe }}$ group. (C) Purification of proteins with flag tags and detection by Western blot assay. Bands were detected in the HMGN2-flag group and FLAG-BAP group using an anti-DDDDK antibody, while no bands were detected in the control group. FLAG-BAP: Amino-terminal FLAG-BAP fusion protein, 49.3kDa. Control: nuclear protein extracts of oeCON group; HMGN2-flag: a purified protein with flag tag from the HMGN2-flag ${ }^{\text {oe }}$ group. Data were presented as the mean $\pm \mathrm{SD} .{ }^{* *} \mathrm{P}<0.001$.

groups were analyzed through the Kruskal-Wallis H test.

\section{Results}

\section{HMGN2 protein was overexpressed and purified}

We overexpressed HMGN2 in 293FT cells, and the infection efficiency confirmed by RT-qPCR. RT-qPCR demonstrated that the level of HMGN2-mRNA expression in the HMGN2-flag ${ }^{\text {oe }}$ group significantly exceeded that of the oeCON group (Figure 1A). Proteins were further purified with flag tags and identified by Western blot assay. HMGN2 was detected in purified protein using an antiHMGN2 antibody (Figure 1B). Bands were detected in the
HMGN2-flag group and the FLAG-BAP group using an anti-DDDDK antibody, while no bands were detected in the Control group (Figure 1C).

\section{Exogenous HMGN2 transport into OS cells and nuclei}

OS cells were treated with purified HMGN2-flag protein at a concentration of $10 \mu \mathrm{g} / \mathrm{mL}$ for 12 or 24 hours, and the cellular location of exogenous protein was detected by immunocytochemistry. The results showed that HMGN2 expressed by eukaryotic cells could be transported into the nucleus of OS cells after 24 hours of treatment (Figure 2A). Next, OS cells were treated with different concentrations 


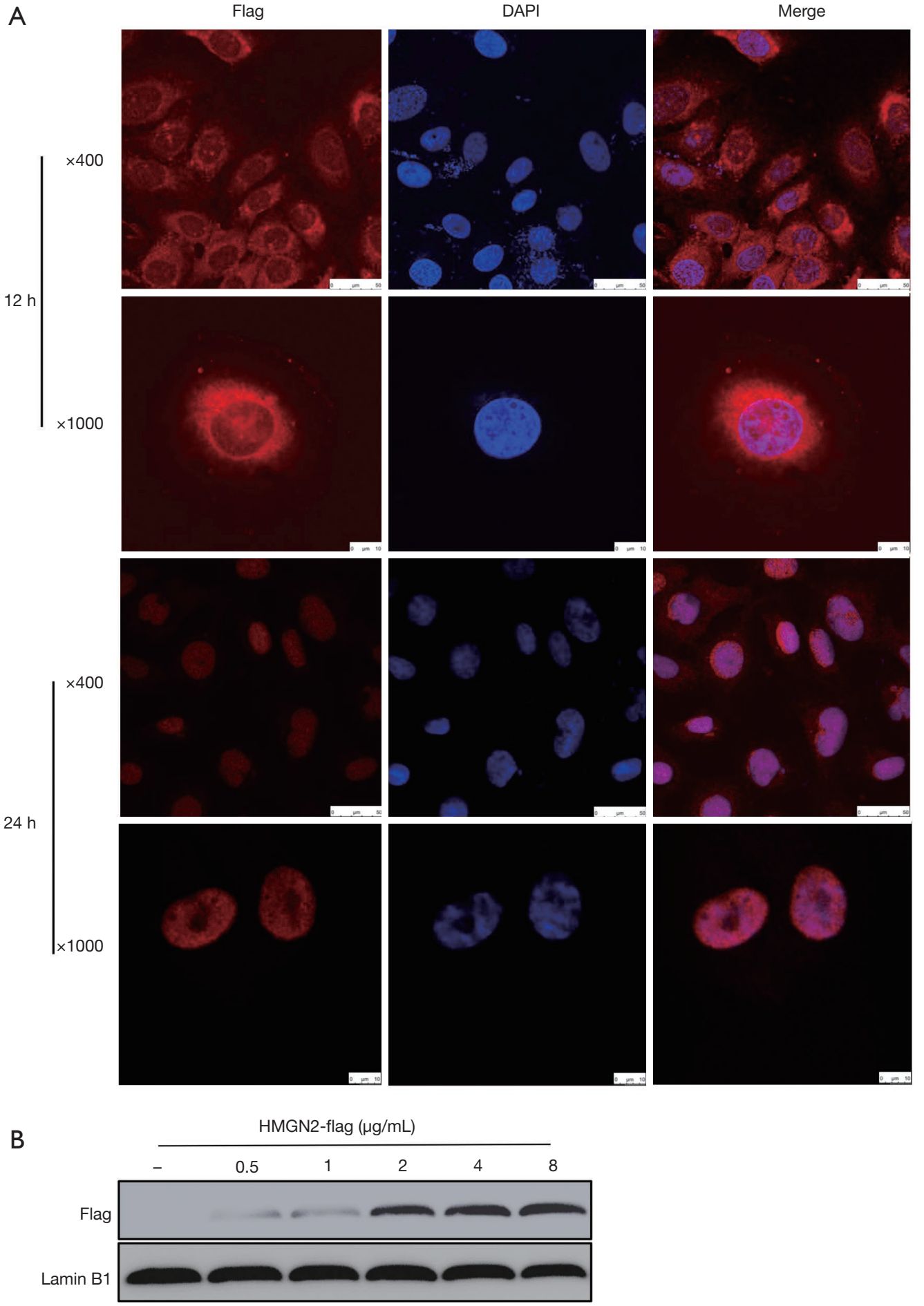

Figure 2 Exogenous HMGN2 transported into osteosarcoma cells and nuclei. (A) OS cells were treated with purified HMGN2-flag protein for 12 or 24 hours, and the cellular location of the exogenous protein was detected by immunocytochemistry. After 24 hours of treatment, HMGN2 could be transported into the nucleus of OS cells. (B) OS cells were treated with different concentrations of HMGN2-flag (0.5, $1,2,4,8 \mu \mathrm{g} / \mathrm{mL}$ ), the nuclear protein was extracted and the level of HMGN2-flag in the nucleus of each treatment group was detected by Western blot assay. The results showed a higher HMGN2-flag level of OS cells as the concentration of HMGN2-flag in the medium increased, and the level did not increase after the extracellular HMGN2-flag concentration exceeded $2 \mu \mathrm{g} / \mathrm{mL}$. 
A UT
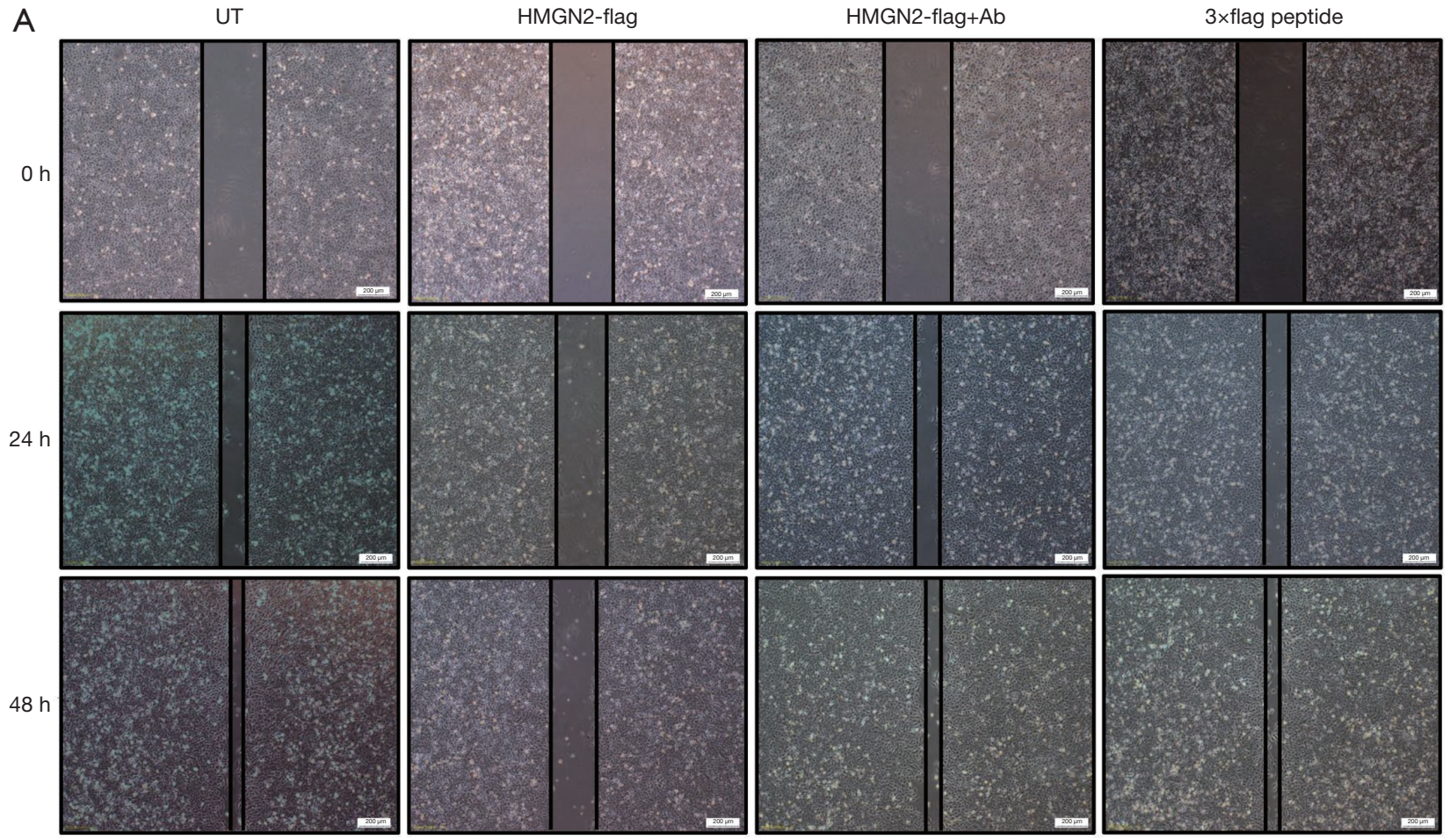

B

C

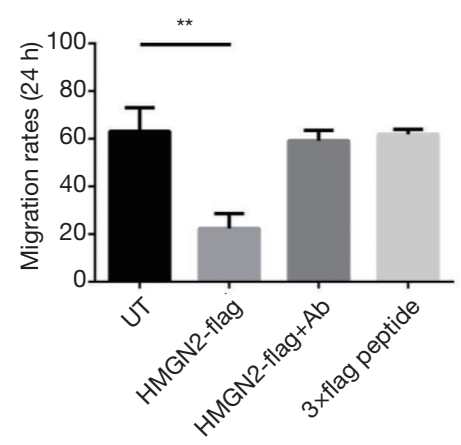

D

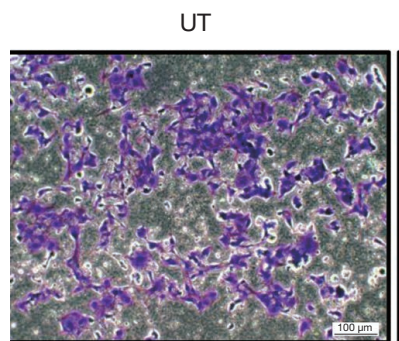

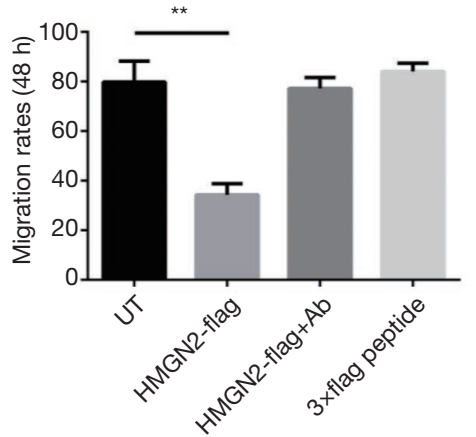

HMGN2-flag

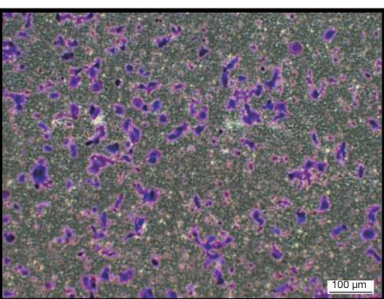

HMGN2-flag+Ab

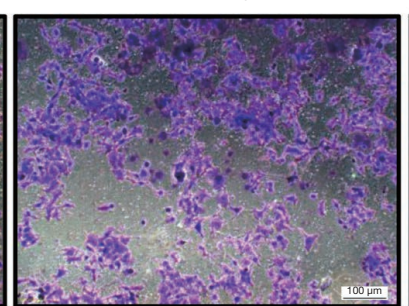

E

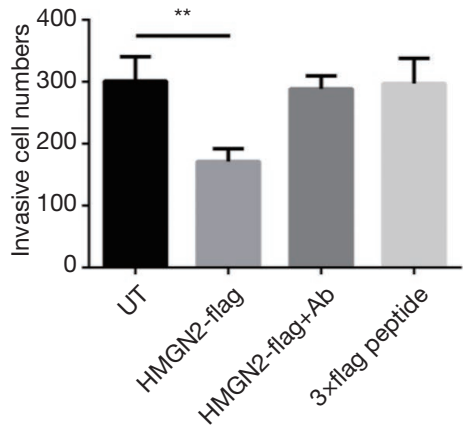

Figure 3 Differences in cell migration and invasion between each group were detected by wound healing and transwell assays. (A,B,C) Wound healing assay showed that the migration capacity of OS cells in the HMGN2-flag group was markedly lower than those in the untreated (UT), HMGN2-flag+Ab and 3×flag peptide groups. UT, untreated; HMGN2-flag, $2 \mu \mathrm{g} / \mathrm{mL}$ purified protein; HMGN2-flag+Ab, $2 \mu \mathrm{g} / \mathrm{mL}$ purified protein $+10 \mu \mathrm{g} / \mathrm{mL}$ Ab; $3 \times f l a g$ peptide, $0.55 \mu \mathrm{g} / \mathrm{mL} 3 \times$ flag peptide (same number of moles as HMGN2-flag). (D,E) Transwell invasion assay of each group. The invasion of OS cells in the HMGN2-flag group was markedly decreased as compared with the UT, HMGN2-flag+Ab and $3 \times$ flag peptide groups. Data were presented as the mean $\pm \mathrm{SD}$. ${ }^{* *} \mathrm{P}<0.01$. 

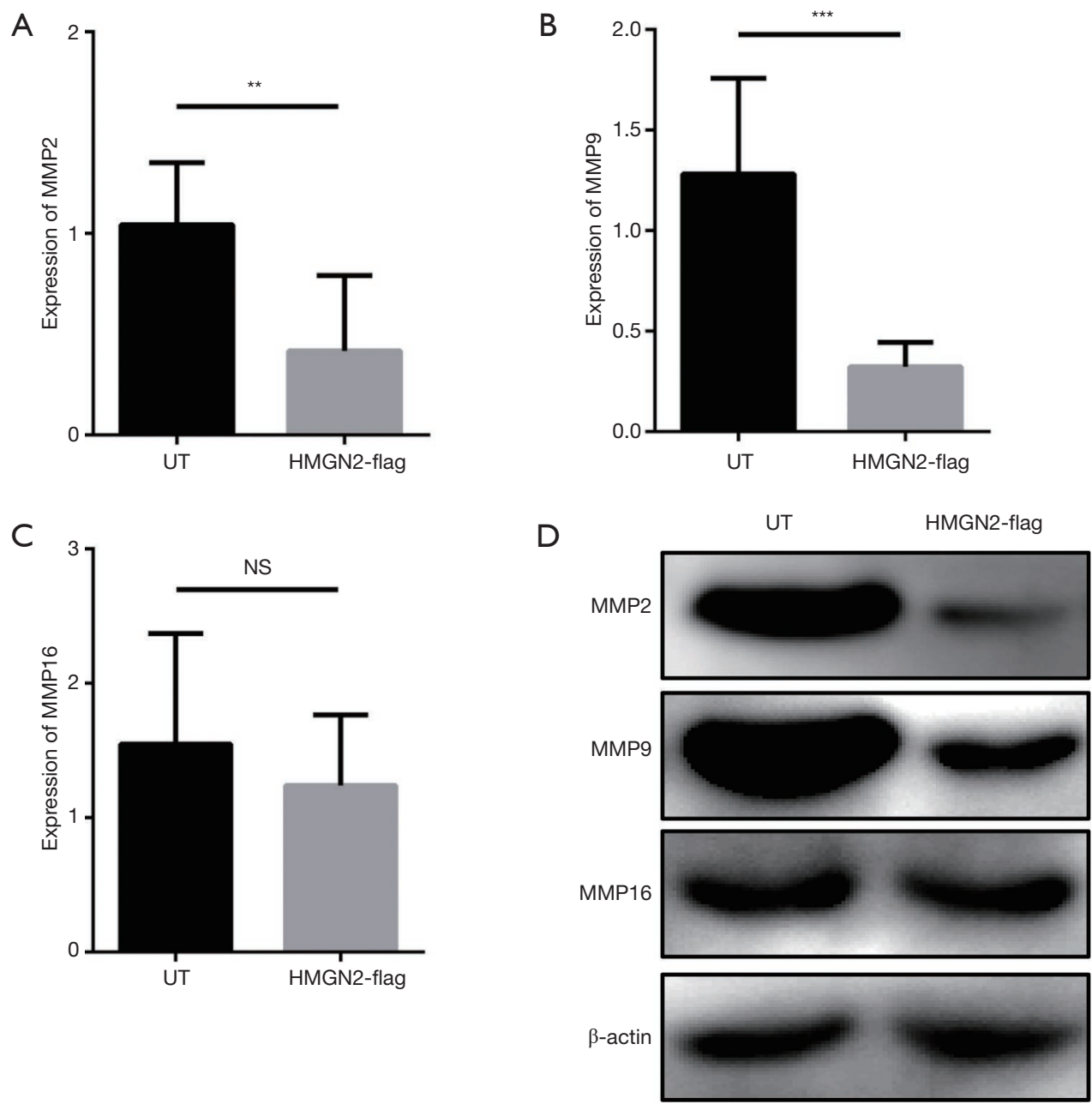

Figure 4 Exogenous HMGN2 inhibits the migration and invasion of osteosarcoma by decreasing MMP2 and MMP9. (A) The expression level of MMP2-mRNA was detected by RT-qPCR. The results showed that the expression level of MMP2-mRNA in the HMGN2-flag group was significantly lower than in the UT group. (B) The expression level of MMP9-mRNA in the HMGN2-flag group was significantly lower than in the UT group. (C) There was no significant difference between the expression levels of MMP16 in the UT and HMGN2flag groups. (D) The expression levels of proteins were detected by Western blot. The results showed that the expression levels of MMP2 and MMP9 proteins in the HMGN2-flag group were lower than those in the UT group. There was no significant difference between the expression levels of MMP16 protein in the UT and HMGN2-flag groups. Data were presented as the mean $\pm \mathrm{SD}$. ${ }^{* *} \mathrm{P}<0.01,{ }^{* * *} \mathrm{P}<0.001$.

of purified HMGN2-flag $(0.5,1,2,4,8 \mu \mathrm{g} / \mathrm{mL})$ for 24 hours, the nuclear protein was then extracted, and the level of HMGN2-flag in the nuclei of each treatment group was detected by Western blot assay. The results showed a higher HMGN2-flag level of OS cells as the concentration of HMGN2-flag in the medium increased, and the level did not increase after the extracellular HMGN2-flag concentration exceeded $2 \mu \mathrm{g} / \mathrm{mL}$ (Figure $2 B$ ).

\section{Exogenous HMGN2 inhibits migration and invasion of OS cells}

According to the wound healing assay, the rate of OS cell migration in the HMGN2-flag group was significantly lower than in the untreated (UT), HMGN2-flag+Ab, and $3 \times f l a g$ peptide groups (Figure $3 A, B, C$ ). Transwell invasion assay of each group showed the invasion rate of OS cells in the HMGN2-flag group was markedly decreased when 
compared with the UT, HMGN2-flag+Ab, and $3 \times$ flag peptide groups (Figure 3D,E).

\section{Exogenous HMGN2 regulates the expression of MMP2 and MMP9}

There is currently no research to explain the mechanism of the anti-tumor functions of HMGN2. MMPs were chosen for analysis because MMPs are regulated by other HMGN family members and are associated with tumor metastasis (19-22). The expression levels of MMP2, MMP9, and MMP16 in each group were detected by RT-qPCR and Western blot. The results of RT-qPCR (Figure $4 A, B, C$ ) and Western blot (Figure 4D) showed that the expression levels of MMP2 and MMP9 in the HMGN2-flag group were significantly lower than in the UT group, and there was no significant difference in the expression level of MMP16 between the UT and HMGN2-flag groups.

\section{Discussion}

About $20 \%$ of patients with OS have metastases at the time of initial diagnosis, and it has been found that $25-50 \%$ of patients subsequently metastasize even if they are not found to have metastasis initially (23). Chemotherapy and surgical intervention are the main methods used to treat OS patients. Nevertheless, in most cases, pulmonary metastases eventually develop, and patients succumb to OS despite these medical interventions (24-26). About $90 \%$ of patients with OS will have metastasis or recurrence, even after surgical removal of the primary tumor (27). It is, therefore, vital that new and safe treatments are developed for OS patients. Some antitumor factors of OS have been found. Wei $\mathrm{H}$ et al. demonstrated that a nanodrug consisting of doxorubicin and exosome derived from mesenchymal stem cells could be used as an excellent chemotherapeutic drug for treatment of OS in vitro (28). Zhong et al. found that Dynasore suppresses cell proliferation, migration, and invasion and enhances the antitumor capacity of cisplatin via STAT3 pathway in OS (29). Xia et al. suggest that downregulation of RAC2 by small interfering RNA restrains the progression of OS by suppressing the Wnt signaling pathway (30).

The high mobility group (HMG), which plays an important role in tumor metastasis, includes HMGA, HMGB, and HMGN $(31,32)$. HMGN is a protein located in the nucleus that is expressed only in eukaryotes. The HMGN protein contains a nucleosome binding domain
(NBD) that specifically binds to nucleosomes and activates transcription $(33,34)$. HMGN2 functions to stabilize nucleosome morphology and regulate gene transcription. The N-terminus of NBD binds to histones, while the C-terminus binds to DNA and regulates transcription (35). Early studies have shown that HMGN2 is a non-histone nucleoprotein that is widely expressed in cells of vertebrates and invertebrates $(34,36)$. HMGN2 increases the entanglement angle of DNA on nucleosomes and promotes nucleocapsid structural remodeling (37). HMGN2-deficient DT40 cells are sensitive to ultraviolet light and increase the apoptotic rate of cells, suggesting that HMGN2 plays a role in DNA damage repair (38). Abnormal expression of HMGN2 is associated with multiple tumors (10-15). HMGN2 has been shown to impede growth in oral squamous cell carcinoma cell line Tca8113, as well as bringing about apoptosis (16). Fan et al. proved that recombinant human HMGN2 could significantly inhibit the proliferation and migration of breast cancer cells, which increased the apoptosis of breast cancer cells and exerted anti-breast cancer effects (39). $\gamma \delta \mathrm{T}$ cells function in the regulation of T-cell activation in cancer and have been identified as a novel target for cancer immunotherapy, Chen et al. found that HMGN2 is an antitumor effector molecule of $\gamma \delta \mathrm{T}$ cells (40). The results of $\mathrm{Li}$ et al. demonstrated that the expression of HMGN2 was increased in the majority of tumor cell lines, particularly MDA-MB-468 and THP1 cells, suggesting that HMGN2 may serve an important function in the growth and metastasis of tumor cells (41). Our previous studies have shown that overexpression of HMGN2 by lentivirus infection in OS cells inhibits the metastasis of cells (17). However, lentiviruses are not currently accepted in the treatment of humans.

IL-2 stimulates the release of HMGN2 from human peripheral blood mononuclear cells $(42,43)$. Tongue squamous cell carcinoma antigen stimulates the release of HMGN2 by $\mathrm{CD}^{+} \mathrm{T}$ lymphocytes, and the released HMGN2 enters the tumor cells and localizes in the nucleus, exerting the killing effect of tumor cells (18). To explore whether exogenous HMGN2 enters OS cells, we purified HMGN2-flag fusion protein and treated OS cells with this fusion protein and detected protein localization by immunocytochemistry (ICC). The results showed that exogenous HMGN2 entered OS cells and eventually located in the nucleus. To understand whether exogenous HMGN2 inhibits OS cell metastasis, we treated OS cells with purified HMGN2 protein and observed differences in the migration and invasion rates of OS cells by wound 
healing assay and transwell invasion assay. Our results showed that the exogenous HMGN2 entered OS cells and inhibited their migration and invasion. These results provide new ideas for research into anti-tumor treatments.

Studies have been carried out which highlight the antitumor activity of HMGN2. A tumor metastasis suppression and tumor targeting peptide study have shown that HMGN2 could offer a potential target in tumor metastasis treatment. The researchers extracted a 21-amino acid peptide from the bovine liver, named invasion inhibitor 2 (IIF2), which was identified to be consistent with the C-terminal fragment of HMGN2 $(44,45)$. This peptide inhibited multiple tumor cell metastasis in vitro (45-47). Animal studies have shown that the simultaneous injection of IIF2 and lung cancer cells into the tail vein of mice reduces metastasis by $50-60 \%$ compared with the control group(45). IIF2 combined with albumin to improve its stability and reduced metastasis formation by $86 \%(46,48)$. Porkka et al. found that HMGN2 is also involved in tumor angiogenesis (42). However, there is currently no research to explain the mechanism of the anti-tumor functions of HMGN2.

Activation of MMPs has been studied in relation to nearly all forms of human cancer, and a close relationship with increased invasion and metastasis, advanced tumor stage, and poor survival time has been identified (49). HMGN2 is a member of the HMGN family. Some previous studies have reported that the other members of the HMGN family, like HMGN5, are associated with metastasis of various tumors $(19,20,22,50,51)$. There was evidence found to suggest HMGNs regulate MMPs expression, and take up a vital role in tumor metastasis (19-22). At the beginning of our research, we speculated that HMGN2 might act as an anti-tumor factor by down-regulating MMPs. The results of our study showed that exogenous HMGN2 regulates MMP2 and MMP9 expression and plays a role in inhibiting the migration and invasion of OS cells.

In this study, we first reported the anti-tumor effects of exogenous HMGN2 in OS cells. However, this is limited research due to the lack of in vivo assay. For our next step, we plan to simultaneously inject HMGN2 and OS cells into the tail vein of nude mice to further determine the antimetastatic effects of exogenous HMGN2.

\section{Conclusions}

In our previous study, we confirmed that HMGN2 overexpression has an anti-tumor effect on human OS cell lines. In this study, we found that exogenous HMGN2 enters OS cells and regulates the expression of MMP2 and MMP9 in OS cells, ultimately contributing to the inhibition of OS cell migration and invasion. These results provide new ideas for future research into the development of antitumor drugs for the treatment of OS.

\section{Acknowledgments}

Funding: This work was supported by the Shanghai Science and Technology Committee of China (No. 16140901800) and National Natural Science Foundation of China (No. U1603118).

\section{Footnote}

Conflicts of Interest: All authors have completed the ICMJE uniform disclosure form (available at http://dx.doi. org/10.21037/tcr.2020.02.25). The authors have no conflicts of interest to declare.

Ethical Statement: The authors are accountable for all aspects of the work in ensuring that questions related to the accuracy or integrity of any part of the work are appropriately investigated and resolved. The study was conducted in accordance with the Declaration of Helsinki (as revised in 2013). Institutional ethical approval and informed consent were waived.

Open Access Statement: This is an Open Access article distributed in accordance with the Creative Commons Attribution-NonCommercial-NoDerivs 4.0 International License (CC BY-NC-ND 4.0), which permits the noncommercial replication and distribution of the article with the strict proviso that no changes or edits are made and the original work is properly cited (including links to both the formal publication through the relevant DOI and the license). See: https://creativecommons.org/licenses/by-nc-nd/4.0/.

\section{References}

1. ESMO/European Sarcoma Network Working Group. Bone sarcomas: ESMO Clinical Practice Guidelines for diagnosis, treatment and follow-up. Ann Oncol 2012;23 Suppl 7:vii100-9.

2. Jo VY, Fletcher CD. WHO classification of soft tissue tumours: an update based on the 2013 (4th) edition. Pathology 2014;46:95-104. 
3. Gordon N, Kleinerman ES. Aerosol therapy for the treatment of osteosarcoma lung metastases: targeting the Fas/FasL pathway and rationale for the use of gemcitabine. J Aerosol Med Pulm Drug Deliv 2010;23:189-96.

4. Allison DC, Carney SC, Ahlmann ER, et al. A metaanalysis of osteosarcoma outcomes in the modern medical era. Sarcoma 2012;2012:704872.

5. Jaffe N, Puri A, Gelderblom H. Osteosarcoma: evolution of treatment paradigms. Sarcoma 2013;2013:203531.

6. Wittig JC, Bickels J, Priebat D, et al. Osteosarcoma: a multidisciplinary approach to diagnosis and treatment. Am Fam Physician 2002;65:1123-32.

7. Meyers PA, Heller G, Healey JH, et al. Osteogenic sarcoma with clinically detectable metastasis at initial presentation. J Clin Oncol 1993;11:449-53.

8. Tsuchiya H, Kanazawa Y, Abdel-Wanis ME, et al. Effect of timing of pulmonary metastases identification on prognosis of patients with osteosarcoma: the Japanese Musculoskeletal Oncology Group study. J Clin Oncol 2002;20:3470-7.

9. Kager L, Zoubek A, Potschger U, et al. Primary metastatic osteosarcoma: presentation and outcome of patients treated on neoadjuvant Cooperative Osteosarcoma Study Group protocols. J Clin Oncol 2003;21:2011-8.

10. Srikantha T, Landsman D, Bustin M. Retropseudogenes for human chromosomal protein HMG-17. J Mol Biol 1987;197:405-13.

11. Spieker N, Beitsma M, van Sluis P, et al. An integrated $5-\mathrm{Mb}$ physical, genetic, and radiation hybrid map of a 1 p36.1 region implicated in neuroblastoma pathogenesis. Genes Chromosomes Cancer 2000;27:143-52.

12. Fiorillo AA, Medler TR, Feeney YB, et al. HMGN2 inducibly binds a novel transactivation domain in nuclear PRLr to coordinate Stat5a-mediated transcription. Mol Endocrinol 2011;25:1550-64.

13. Medler TR, Craig JM, Fiorillo AA, et al. HDAC6 Deacetylates HMGN2 to Regulate Stat5a Activity and Breast Cancer Growth. Mol Cancer Res 2016;14:994-1008.

14. Schauwecker SM, Kim JJ, Licht JD, et al. Histone H1 and Chromosomal Protein HMGN2 Regulate Prolactininduced STAT5 Transcription Factor Recruitment and Function in Breast Cancer Cells. J Biol Chem 2017;292:2237-54.

15. Wei D, Zhang P, Zhou M, et al. HMGN2 protein inhibits the growth of infected T24 cells in vitro. J Cancer Res Ther 2014;10:299-304.

16. Hu A, Dong X, Liu X, et al. Nucleosome-binding protein HMGN2 exhibits antitumor activity in oral squamous cell carcinoma. Oncol Lett 2014;7:115-20.

17. Liang G, Xu E, Yang C, et al. Nucleosome-binding protein HMGN2 exhibits antitumor activity in human $\mathrm{SaO} 2$ and U2-OS osteosarcoma cell lines. Oncol Rep 2015;33:1300-6.

18. Su L, Hu A, Luo Y, et al. HMGN2, a new anti-tumor effector molecule of CD8(+) T cells. Mol Cancer 2014;13:178.

19. Zhou X, Yuan B, Yuan W, et al. The expression and clinical significance of high mobility group nucleosome binding domain 5 in human osteosarcoma. Tumour Biol 2014;35:6539-47.

20. Weng M, Song F, Chen J, et al. The high-mobility group nucleosome-binding domain 5 is highly expressed in breast cancer and promotes the proliferation and invasion of breast cancer cells. Tumour Biol 2015;36:959-66.

21. Wahafu W, He ZS, Zhang XY, et al. The nucleosome binding protein NSBP1 is highly expressed in human bladder cancer and promotes the proliferation and invasion of bladder cancer cells. Tumour Biol 2011;32:931-9.

22. Ji SQ, Yao L, Zhang XY, et al. Knockdown of the nucleosome binding protein 1 inhibits the growth and invasion of clear cell renal cell carcinoma cells in vitro and in vivo. J Exp Clin Cancer Res 2012;31:22.

23. Arlt MJ, Banke IJ, Walters DK, et al. LacZ transgene expression in the subcutaneous Dunn/LM8 osteosarcoma mouse model allows for the identification of micrometastasis. J Orthop Res 2011;29:938-46.

24. Yang J, Zhang W. New molecular insights into osteosarcoma targeted therapy. Curr Opin Oncol 2013;25:398-406.

25. Botter SM, Neri D, Fuchs B. Recent advances in osteosarcoma. Curr Opin Pharmacol 2014;16:15-23.

26. He JP, Hao Y, Wang XL, et al. Review of the molecular pathogenesis of osteosarcoma. Asian Pac J Cancer Prev 2014;15:5967-76

27. Wong IH, Chan AT, Johnson PJ. Quantitative analysis of circulating tumor cells in peripheral blood of osteosarcoma patients using osteoblast-specific messenger RNA markers: a pilot study. Clin Cancer Res 2000;6:2183-8.

28. Wei H, Chen J, Wang S, et al. A Nanodrug Consisting Of Doxorubicin And Exosome Derived From Mesenchymal Stem Cells For Osteosarcoma Treatment In Vitro. Int J Nanomedicine 2019;14:8603-10.

29. Zhong B, Shi D, Wu F, et al. Dynasore suppresses cell proliferation, migration, and invasion and enhances the antitumor capacity of cisplatin via STAT3 pathway in osteosarcoma. Cell Death Dis 2019;10:687.

30. Xia P, Gao X, Shao L, et al. Down-regulation of RAC2 
by small interfering RNA restrains the progression of osteosarcoma by suppressing the Wnt signaling pathway. Int J Biol Macromol 2019;137:1221-31.

31. Reeves R. Molecular biology of HMGA proteins: hubs of nuclear function. Gene 2001;277:63-81.

32. Ohmori H, Luo Y, Kuniyasu H. Non-histone nuclear factor HMGB1 as a therapeutic target in colorectal cancer. Expert Opin Ther Targets 2011;15:183-93.

33. Bustin M, Reeves R. High-mobility-group chromosomal proteins: architectural components that facilitate chromatin function. Prog Nucleic Acid Res Mol Biol 1996;54:35-100.

34. Bustin M. Regulation of DNA-dependent activities by the functional motifs of the high-mobility-group chromosomal proteins. Mol Cell Biol 1999;19:5237-46.

35. Kato $\mathrm{H}$, van Ingen $\mathrm{H}$, Zhou BR, et al. Architecture of the high mobility group nucleosomal protein 2-nucleosome complex as revealed by methyl-based NMR. Proc Natl Acad Sci U S A 2011;108:12283-8.

36. Postnikov YV, Herrera JE, Hock R, et al. Clusters of nucleosomes containing chromosomal protein HMG-17 in chromatin. J Mol Biol 1997;274:454-65.

37. Shimahara H, Hirano T, Ohya K, et al. Nucleosome structural changes induced by binding of non-histone chromosomal proteins HMGN1 and HMGN2. FEBS Open Bio 2013;3:184-91.

38. Subramanian M, Gonzalez RW, Patil H, et al. The nucleosome-binding protein HMGN2 modulates global genome repair. FEBS J 2009;276:6646-57.

39. Fan B, Shi S, Shen X, et al. Effect of HMGN2 on proliferation and apoptosis of MCF-7 breast cancer cells. Oncol Lett 2019;17:1160-6.

40. Chen J, Fan Y, Cui B, et al. HMGN2: An Antitumor Effector Molecule of gammadeltaT Cells. J Immunother 2018;41:118-24.

41. Li Q, Chen J, Li X, et al. Increased expression of highmobility group nucleosomal-binding domain 2 protein in

Cite this article as: $\mathrm{Xu}$ E, Jiang $\mathrm{H}$, Lin T, Meng Y, Ma X, Yin J, Ma J, Zhou X. Exogenous HMGN2 inhibits the migration and invasion of osteosarcoma cell lines. Transl Cancer Res 2020;9(3):1795-1805. doi: 10.21037/tcr.2020.02.25 various tumor cell lines. Oncol Lett 2018;15:4517-22.

42. Porkka K, Laakkonen P, Hoffman JA, et al. A fragment of the HMGN2 protein homes to the nuclei of tumor cells and tumor endothelial cells in vivo. Proc Natl Acad Sci U S A 2002;99:7444-9.

43. Feng Y, Huang N, Wu Q, et al. HMGN2: a novel antimicrobial effector molecule of human mononuclear leukocytes? J Leukoc Biol 2005;78:1136-41.

44. Isoai A, Giga-Hama Y, Shinkai K, et al. Purification and characterization of tumor invasion-inhibiting factors. Jpn J Cancer Res 1990;81:909-14.

45. Isoai A, Giga-Hama Y, Shinkai K, et al. Tumor invasioninhibiting factor 2: primary structure and inhibitory effect on invasion in vitro and pulmonary metastasis of tumor cells. Cancer Res 1992;52:1422-6.

46. Isoai A, Goto-Tsukamoto H, Murakami K, et al. A potent anti-metastatic activity of tumor invasion-inhibiting factor-2 and albumin conjugate. Biochem Biophys Res Commun 1993;192:7-14.

47. Han ZG, Jiang WG, Hiscox S, et al. Inhibition of motility and invasion of human lung cancer cells by invasion inhibiting factor 2. Surg Oncol 1996;5:77-84.

48. Isoai A, Goto-Tsukamoto H, Yamori T, et al. Inhibitory effects of tumor invasion-inhibiting factor 2 and its conjugate on disseminating tumor cells. Cancer Res 1994;54:1264-70.

49. Egeblad M, Werb Z. New functions for the matrix metalloproteinases in cancer progression. Nat Rev Cancer 2002;2:161-74.

50. Gan Y, Tan J, Yang J, et al. Knockdown of HMGN5 suppresses the viability and invasion of human urothelial bladder cancer 5637 cells in vitro and in vivo. Med Oncol 2015;32:136.

51. Wei P, Qiao B, Li Q, et al. microRNA-340 Suppresses Tumorigenic Potential of Prostate Cancer Cells by Targeting High-Mobility Group Nucleosome-Binding Domain 5. DNA Cell Biol 2016;35:33-43. 